\title{
Câncer de Mama Associado à Gravidez: Um Estudo Caso/Controle
}

\author{
Breast Cancer Associated With Pregnancy: A Case Control Study \\ Juvenal Mottola Junior, Anastasio Berrettini Junior, Cídia Mazzoccato, Fábio Laginha, \\ César Eduardo Fernandes, José Antonio Marques
}

\begin{abstract}
RESUMO
Objetivo: relatar série de 15 casos de câncer de mama associado à gravidez e comparar com um grupo controle de pacientes jovens com carcinoma ductal invasivo da mama, avaliando o estadiamento clinico, o comprometimento linfonodal axilar, o grau nuclear, o grau histológico e os receptores hormonais de estrógeno e progesterona.

Métodos: foi realizado estudo retrospectivo de 15 casos de pacientes com câncer de mama associado à gravidez, atendidas no setor de Mastologia do Centro de Referência da Saúde da Mulher, Hospital Pérola Byington, em São Paulo, durante o período de setembro de 1996 a abril de 2001, designando como base principal do estudo a avaliação do estadiamento clínico, a época do diagnóstico e o comprometimento axilar. Também, foram analisados a faixa etária, paridade, tipo histológico, tratamento realizado, características histológicas quanto ao grau nuclear e grau histológico, e a presença de receptores hormonais nos tumores diagnosticados. Comparou-se este grupo com um grupo controle de pacientes jovens com câncer de mama.

Resultados: verificou-se que 7 pacientes com câncer de mama associado à gravidez $(46,7 \%)$ encontravam-se com doença localmente avançada (estádio clínico IIIA e IIIB) e 3 pacientes (20\%) apresentavam doença disseminada no momento do diagnóstico. As pacientes apresentaram em média 2,4 linfonodos axilares comprometidos, sendo que apenas uma paciente $(6,6 \%)$ não apresentava comprometimento linfonodal axilar. Com relação à época do diagnóstico, 40\% dos cânceres foram diagnosticados durante a lactação, 46,7\% durante o terceiro trimestre e 13,3\% no segundo trimestre. Comparou-se este grupo de pacientes grávidas com um grupo controle de pacientes, com a mesma média etária, não grávidas, portadoras de carcinoma invasivo de mama analisando o estadiamento clínico, o comprometimento linfonodal axilar, grau nuclear, grau histológico e os receptores hormonais de estrógeno e progesterona. Houve diferença estatisticamente significante $(p=0,0022)$ para o estadiamento clínico e para o comprometimento linfonodal axilar ( $p=0,0017)$, não havendo diferença estatisticamente significativa para os demais parâmetros analisados.

Conclusão: o câncer de mama associado à gravidez mantém-se como neoplasia de mau prognóstico, não havendo diferença quando se compara com pacientes não grávidas para a mesma média de faixa etária, sendo que o fator determinante na sobrevida é o estádio clínico avançado no momento do diagnóstico.
\end{abstract}

PALAVRAS-CHAVE: Mama: câncer. Complicações da gravidez. Quimioterapia.

Centro de Referência da Saúde da Mulher, Hospital Pérola Byington, São Paulo.

Correspondência:

Juvenal Mottola Junior

Rua Dr. Zuquim 449, cj 51/52 - Santana

02035-010 - São Paulo - SP

e-mail: jmottola@uol.com.br
Introdução

As décadas de 1970 e 1980 representaram o surgimento do movimento feminista e, progressivamente, as mulheres foram adquirindo maior 
independência tanto profissionalmente quanto em relação à família e, dentro do modelo econômico que se instalou, as mulheres conquistaram seu espaço na sociedade. Esta transformação feminina refletiu na medicina contemporânea, especialmente na obstetrícia, quando cada vez mais mulheres estão retardando a gravidez para as $3^{a}$ e $4^{\text {a década de suas vidas }}{ }^{1,2}$. Conseqüentemente, doenças que comumente acometem mulheres em idade adulta associam-se às gestações mais tardias. Paralelamente, o câncer de mama está sendo diagnosticado em mulheres em idade mais jovem $^{2}$, não se sabendo se isso é devido ao diagnóstico mais precoce pelo avanço da tecnologia médica, conscientização feminina e programas de rastreamento ou se a história natural da doença está se modificando pelas alterações ambientais e pela exposição a agentes potencialmente cancerígenos.

Aceita-se que câncer de mama associado à gravidez é todo câncer de mama diagnosticado durante a gravidez ou até um ano após o parto ${ }^{1}$. O câncer de mama representa 0,2 a 3,8\% de todos os cânceres que ocorrem na gestação. Ocorre numa relação de $1 / 3.000$ a $1 / 10.000$ gestações $^{2}$ dependendo do país estudado, sendo que algumas séries atuais apresentam associação de 1/2.000 gestações. Apresenta-se como a segunda causa de neoplasia associada à gravidez, ultrapassada apenas pelo câncer de colo uterino. Nos Estados Unidos da América do Norte, a estimativa para o ano de 2000 foi de 3500 novos casos $^{1-3}$.

Os primeiros relatos, há mais de 100 anos, sobre câncer de mama associado à gravidez demonstravam um prognóstico sombrio a respeito desta associação. Os primeiros médicos a publicarem e a estudarem câncer de mama associado à gravidez foram Kilgore e Bloodgood ${ }^{4}$, que apresentaram uma série de casos no início do século XX, e após cinco anos de acompanhamento, todas as pacientes evoluíram para óbito. Em 1954, White ${ }^{5}$ relatou taxa de sobrevida em 5 anos de 17\%; Haagensen e Stout ${ }^{6}$ relataram taxa de sobrevida global em 5 anos de apenas 8,6\%.

O primeiro relato otimismo é de 1937. Neste Harrington ${ }^{7}$ relatou taxa de sobrevida global de $61 \%$ nas pacientes com linfonodos axilares negativos. Sabe-se que o comprometimento axilar era, e continua sendo, comum em mulheres com câncer de mama e grávidas ou diagnosticado até um ano após o parto.

Quatro artigos foram publicados na década de $70^{3,8-10}$ nos quais encontravam-se taxas de linfonodos positivos entre 56 e $81 \%$. Poucos estudos tentaram correlacionar estes percentuais no contexto da idade das pacientes, época do diagnós- tico e semelhança demográfica, comparando com grupo de mulheres não grávidas.

Algumas séries modernas de tipo caso/controle ${ }^{10,11}$ indicam que a gravidez é um fator de risco que independe do estádio por ocasião do diagnóstico. De maneira oposta, outros relatos indicam que a gravidez está associada a estádio mais avançado por ocasião do diagnóstico, porém, quando comparadas às mulheres não grávidas com o mesmo estádio clínico, não se verificaram diferenças significativas com relação à sobrevida e intervalo livre de doença ${ }^{12-14}$.

O objetivo deste estudo foi relatar uma série de 15 casos de câncer de mama associado à gravidez e comparar com um grupo controle de 31 pacientes não grávidas com carcinoma ductal invasivo da mama, avaliando o estadiamento clínico, o comprometimento linfonodal axilar, aspectos histológicos como o grau nuclear e o grau histológico e os receptores hormonais de estrógeno e de progesterona.

\section{Pacientes e Métodos}

Foi realizado estudo retrospectivo de 15 pacientes com câncer de mama associado à gravidez, durante o período de setembro de 1996 a abril de 2001, atendidas no Setor de Mastologia do Centro de Referência da Saúde da Mulher, Hospital Pérola Byington, São Paulo.

Todas as pacientes foram submetidas a tratamento de acordo com protocolo institucional para as pacientes com câncer de mama associado à gravidez. A indicação cirúrgica seguiu os critérios utilizados para pacientes não grávidas, tornando-se possivel realização de cirurgia conservadora, quando respeitado o período máximo de 24 semanas para realização da radioterapia. Nenhuma paciente foi submetida a tratamento quimioterápico no primeiro trimestre.

Dentre as variáveis estudadas para as pacientes grávidas destacamos o estadiamento clínico, a época do diagnóstico e o comprometimento linfonodal axilar como base fundamental deste estudo. Também, avaliamos a faixa etária, paridade, tipo histológico, tratamento realizado, características histológicas quanto ao grau nuclear e grau histológico e presença de receptores hormonais nos tumores diagnosticados.

O grau do tumor é um fator prognóstico importante, sendo adotada a classificação de ScarffBloom-Richardson (SBR) modificada, em que são analisados a formação de túbulos, atividade mitótica e pleomorfismo nuclear, sendo expressos como graus I, II e III. 
As pacientes grávidas foram comparadas com um grupo controle de pacientes jovens não grávidas com carcinoma ductal invasivo da mama, analisando as mesmas variáveis como o estadiamento clínico, comprometimento linfonodal axilar, o grau nuclear, o grau histológico e os receptores hormonais de estrógeno e progesterona.

No momento do diagnóstico, as pacientes foram estadiadas pelo sistema TNM (UICC), e foram utilizados critérios clínicos e imagenológicos, utilizando-se a radiografia de tórax, ultra-sonografia (USG) pélvica e abdominal e cintilografia óssea.

Apresentamos, também, dados dos recémnascidos, obtidos após avaliação do neonatologista.

\section{Resultados}

A média etária das pacientes grávidas foi de 31,1 , anos variando entre 25 e 40 anos. Com relação à paridade, duas pacientes $(13,3 \%)$ eram primigestas, doze pacientes $(80 \%)$ estavam na $2^{\text {a }}$ e $3^{\text {a }}$ gestação e uma paciente $(6,6 \%)$ era quartigesta. Quanto ao estadiamento clínico (EC), 5 pacientes (33\%) encontravam-se com EC II, 7 pacientes $(46,7 \%)$ no EC III e 3 pacientes (20\%) apresentaram doença disseminada (EC IV) no momento do diagnóstico.

Com relação ao tipo de tratamento, dez pacientes $(66,7 \%)$ foram submetidas a mastectomia radical modificada e para três pacientes $(20 \%)$ a opção foi quadrantectomia com esvaziamento completo axilar. Avaliando-se o grau nuclear (GN), quatro pacientes $(26,7 \%)$ apresentaram GN I, quatro pacientes (26,7\%), GN II, e sete pacientes $(46,6 \%)$, GN III, e, quanto ao grau histológico (GH), duas pacientes $(13,3 \%)$ apresentaram GH I, cinco pacientes (33,3\%), GH II, e 8 pacientes (53,3\%), GH III. Quanto à presença dos receptores hormonais, nove pacientes $(60 \%)$ apresentaram receptor estrogênico (RE) negativo, quatro pacientes $(26,6 \%)$, RE positivo, e em duas pacientes (13,3\%) não foi possivel a avaliação de RE. Analisando o número de linfonodos axilares (LA) acometidos, observouse média de 22,3 LA dissecados, com média de acometimento de 2,4 LA por paciente e 14 pacientes $(93,3 \%)$ apresentaram comprometimento linfonodal axilar; assim, apenas uma paciente apresentou axila negativa.

Como tratamento adjuvante, todas as pacientes foram submetidas à quimioterapia, sendo que sete pacientes $(46,6 \%)$ estavam no período gestacional, tendo sido utilizado esquema quimioterápico com 5-fluorouracil, epirrubicina e ciclofosfamida. Em 10 pacientes $(66,6 \%)$ realizou- se radioterapia e, em um caso, aguardou-se 20 semanas após a realização da quadrantectomia.

O tempo médio de seguimento destas pacientes foi de 23,2 meses, variando de 4 a 60 meses. Quanto a sobrevida livre de doença e sobrevida global, 9 pacientes (60\%) estão vivas e sem doença, 4 pacientes $(26,6 \%)$ estão vivas com doença e 2 pacientes $(13,4 \%)$ foram a óbito.

Dentre as nove pacientes (60\%) que tiveram o diagnóstico realizado durante o período gestacional, duas pacientes abortaram, sendo um espontâneo e outro por opção de tratamento conservador. As sete pacientes $(46,6 \%)$ restantes tiveram recém-nascidos (RN) vivos sem malformações, sendo que dois RN apresentaram icterícia fisiológica. Uma paciente evoluiu para trabalho de parto prematuro, dando à luz na $32^{\mathrm{a}}$ semana de gravidez, sendo que o RN não apresentou malformações.

Das pacientes jovens não grávidas com câncer de mama, a média etária foi de 37,4 anos, variando de 29 a 40 anos, e 23 pacientes $(74,2 \%)$ apresentaram-se com estadiamento clínico I e II e 8 pacientes $(25,8 \%)$ com estadiamentos clínicos III e IV, com diferença estatisticamente significante em relação às pacientes grávidas (Tabela 1). As pacientes grávidas apresentaram maior comprometimento linfonodal axilar, com diferenças significante pelo teste do $\left(\chi^{2}\right)$, com $\mathrm{p}=0,0017$ (Tabela 2).

Tabela 1 - Estadiamento clínico (EC) do câncer de mama em pacientes jovens grávidas e não grávidas.

\begin{tabular}{lrcrc}
\hline & $\begin{array}{r}\text { Pacientes grávidas } \\
(\mathbf{n}=\mathbf{1 5})\end{array}$ & \multicolumn{2}{r}{$\begin{array}{r}\text { Pacientes não grávidas } \\
(\mathbf{n}=\mathbf{3 1 )}\end{array}$} \\
\hline EC I e II & $\mathbf{n}$ & $\%$ & $\mathbf{n}$ & $\%$ \\
EC III e IV & 11 & 26,7 & 23 & 74,2 \\
\hline$\chi^{2 \cdot 9,42 \cdot p=0,0022}$ & & & 8 & 25,8 \\
\hline
\end{tabular}

Tabela 2 - Comprometimento linfonodal axilar (LA) em pacientes jovens grávidas e não grávidas com câncer de mama.

\begin{tabular}{lrrrr}
\hline & \multicolumn{2}{c}{$\begin{array}{c}\text { Pacientes grávidas } \\
(\mathbf{n}=\mathbf{1 5})\end{array}$} & \multicolumn{2}{c}{$\begin{array}{r}\text { Pacientes não grávidas } \\
(\mathbf{n}=\mathbf{3 1})\end{array}$} \\
& $\mathbf{n}$ & $\%$ & $\mathbf{n}$ & $\%$ \\
\hline LA positivo & 14 & 93,3 & 14 & 45,2 \\
LA negativo & 1 & 6,7 & 17 & 54,8 \\
\hline$\chi^{2}: 9,85 ; p=0,0017$ & & & &
\end{tabular}

No que se refere ao grau nuclear, grau histológico e aos receptores hormonais de 
estrógeno e progesterona, não se observou diferença estatisticamente significante entre os dois grupos pelo teste do $\chi^{2}$ e pelo teste de Wilcoxon (Tabelas de 3 a 6).

Tabela 3 - Avaliação do grau nuclear em pacientes jovens grávidas e não grávidas com câncer de mama.

\begin{tabular}{lccrc}
\hline & $\begin{array}{c}\text { Pacientes grávidas } \\
(\mathbf{n}=\mathbf{1 5})\end{array}$ & $\begin{array}{r}\text { Pacientes não grávidas } \\
(\mathbf{n}=\mathbf{3 1})\end{array}$ \\
& $\mathbf{n}$ & $\%$ & $\mathbf{n}$ & $\%$ \\
\hline Grau nuclear I & 4 & 26,7 & 3 & 9,7 \\
Grau nuclear II & 4 & 26,7 & 19 & 61,3 \\
Grau nuclear III & 7 & 46,7 & 9 & 29,0 \\
\hline
\end{tabular}

Teste de Wilcoxon: $(N=3 ; Z=1,069045 ; p=0,285057)$

Tabela 4 - Avaliação do grau histológico em pacientes jovens grávidas e não grávidas com câncer de mama.

\begin{tabular}{lcccc}
\hline & \multicolumn{3}{c}{$\begin{array}{c}\text { Pacientes grávidas } \\
(\mathbf{n}=\mathbf{1 5})\end{array}$} & \multicolumn{2}{c}{$\begin{array}{c}\text { Pacientes não grávidas } \\
(\mathbf{n}=\mathbf{3 1})\end{array}$} \\
& $\mathbf{n}$ & $\%$ & $\mathbf{n}$ & $\%$ \\
\hline Grau histológico I & 2 & 13,3 & 3 & 9,7 \\
Grau histológico II & 5 & 33,3 & 22 & 71,0 \\
Grau histológico III & 8 & 53,3 & 6 & 19,3 \\
\hline
\end{tabular}

Teste de Wilcoxon: $(N=3 ; z=0,534522 ; p=0.592984)$

Tabela 5 - Identificação de receptores de estrogênio (RE) em pacientes jovens grávidas e não grávidas com câncer de mama.

\begin{tabular}{lrcrc}
\hline & $\begin{array}{r}\text { Pacientes grávidas } \\
(\mathbf{n}=\mathbf{1 5})\end{array}$ & $\begin{array}{r}\text { Pacientes não grávidas } \\
(\mathbf{n}=\mathbf{3 1})\end{array}$ \\
\hline RE positivo & 4 & 26,7 & 9 & 29,0 \\
RE negativo & 11 & 73,3 & 22 & 71,0 \\
\hline$\chi^{2 \cdot 0,03 \cdot p=0,8673}$ & & &
\end{tabular}

$\chi^{2}: 0,03 ; p=0,8673$

Tabela 6 - Identificação de receptores de progesterona (RP) em pacientes jovens grávidas e não grávidas com câncer de mama.

\begin{tabular}{lcccc}
\hline & $\begin{array}{r}\text { Pacientes grávidas } \\
(\mathbf{n}=\mathbf{1 5})\end{array}$ & \multicolumn{2}{c}{$\begin{array}{r}\text { Pacientes não grávidas } \\
(\mathbf{n}=\mathbf{3 1})\end{array}$} \\
\hline RP positivo & 4 & 26,7 & $\mathbf{n}$ & $\%$ \\
RP negativo & 11 & 73,3 & 6 & 19,3 \\
\hline
\end{tabular}

$\chi^{2}: 0,23 ; p=0,5730$

\section{Discussão}

A abordagem do câncer de mama associado à gravidez tem se modificado nas últimas duas décadas, sendo que os preceitos de mau prognóstico desta associação advindos dos primeiros relatos de casos há mais de 100 anos, foram ultrapassados.

A paciente grávida com câncer de mama pode apresentar as mesmas queixas clínicas de mulher não grávida. As alterações fisiológicas encontradas na mama gravídica - hipertrofia e ingurgitamento - podem contribuir para a demora no diagnóstico. Nas primeiras semanas de gravidez há um proliferação dos ductos e lóbulos, juntamente com aumento das secreções, assim como ingurgitamento vascular. À medida que a gravidez avança, os ácinos lobulares dilatam e ficam repletos de colostro. Há estudos que relatam uma demora no diagnóstico de 3 meses ou mais em pacientes grávidas ou lactantes ${ }^{14}$. Em nossa série de casos, $40 \%$ dos diagnósticos foram realizados durante o periodo lactacional, sendo que $83 \%$ destas pacientes tiveram assistência pré-natal.

Uma vez suspeitando-se de câncer de mama em mulher grávida ou lactante, ela deverá ser imediatamente avaliada, tomando-se os mesmos procedimentos como se a mesma não estivesse grávida. Dentro da propedêutica complementar, a efetividade da mamografia no diagnóstico do câncer de mama durante a gravidez é controversa. A diminuição na sensibilidade da mamografia pode ser atribuída ao aumento na secreção de líquidos e à perda do conteúdo gorduroso na mama gravídica. Enquanto existem alguns debates a respeito da efetividade e exatidão da mamografia em mulheres grávidas ou lactantes no diagnóstico do câncer de mama, o procedimento poderá ser realizado satisfatoriamente em mulheres grávidas, utilizando-se proteção abdominal ${ }^{14,15}$.

O valor da USG mamária durante a gravidez, diferenciando a presença de tumorações sólidas ou císticas, tem sido relatado em alguns poucos estudos. Apesar de não haver respaldo na literatura científica como sendo exame subsidiário no rastreamento do câncer de mama, a USG mamária está clinicamente indicada no diagnóstico de tumoração mamária na gravidez, por ser método inócuo e sem riscos fetais ${ }^{15}$. Assim como na mulher não grávida, a USG pode ser utilizada para guiar procedimentos invasivos com agulhas.

Ao se encontrar tumoração mamária em mulheres grávidas ou lactantes, a punção biópsia por agulha fina (PAAF) ou por agulha grossa (PBAG) deve ser realizada para conclusão diagnóstica, por se tratar de método eficaz e inócuo. Em um estu- 
do avaliando-se os resultados citológicos de biópsias realizadas durante o ciclo gravídicopuerperal ${ }^{15}$, encontraram-se alterações benignas em $80 \%$ das biópsias. Para se aumentar a exatidão da biópsia, deve-se informar ao patologista que se trata de paciente grávida, pois se pode encontrar altos índices de casos falso-positivos, devido ao aumento das mitoses e da celularidade na mama gravídica ${ }^{8}$.

Ao se discutir o tratamento do câncer de mama associado à gravidez, a conduta deve ser baseada nos mesmos princípios utilizados no tratamento de mulher não grávida, com algumas ressalvas, principalmente no primeiro trimestre de gravidez, pois durante este período a administração de quimioterápicos está associada a teratogênese e abortamentos ${ }^{3}$. A paciente que apresenta câncer de mama localmente avançado ou doença sistêmica no momento do diagnóstico deve ser submetida à terapêutica sistêmica, sendo o tratamento cirúrgico postergado. Quando o câncer de mama é diagnosticado no primeiro trimestre de gravidez, o tratamento preconizado é a mastectomia, seja ela radical se estiver indicada, ou modificada. O tratamento conservador neste período deve ser exceção, e caso se decida por ele, deve-se discutir a interrupção da gravidez, tendo em vista a necessidade de radioterapia complementar, que tem contra-indicação absoluta durante a gravidez. Durante o segundo e terceiro trimestres da gravidez, o tratamento conservador já pode ser considerado, já que o intervalo entre a cirurgia e a realização da radioterapia não deve ultrapassar 24 semanas $^{14}$. Dependendo da idade gestacional, o parto poderá ser antecipado para que a radioterapia possa ser realizada.

O comprometimento linfonodal axilar continua sendo comum em pacientes grávidas e lactantes ${ }^{1-3,11,16}$. Isso se deve ao estádio clínico avançado no momento do diagnóstico e está intimamente relacionado ao prognóstico e intervalo livre de doença. Em nossa série de casos, aproximadamente $70 \%$ apresentavam-se no EC IIIa, IIIb e IV, sendo semelhante aos dados de outras séries de $\operatorname{casos}^{1-3}$, e a distribuição foi diferente do grupo controle.

As indicações para terapia sistêmica em mulheres grávidas ou lactantes obedecem às regras para as mulheres não-gestantes. As alterações fisiológicas da gravidez, incluindo alterações nas funções hepáticas e renais, aumento do volume plasmático e o "terceiro espaço" do líquido amniótico, podem influenciar a distribuição das drogas antineoplásicas ${ }^{9}$. Os efeitos adversos potenciais dos agentes antineoplásicos sobre feto $\mathrm{e}$ o recém-nascido incluem efeitos imediatos, como teratogênese e abortamento, ou efeitos tardios, como retardo de crescimento e disfunção gonádi$\mathrm{ca}^{3}$. Durante o primeiro trimestre, a realização da quimioterapia é contra-indicada, devido ao fato de a organogênese ocorrer durante as primeiras dez semanas de gravide $z^{1,14}$.

A administração de drogas anti-neoplásicas durante o segundo e o terceiro trimestres é relativamente segura, sendo que as drogas que não induziram alterações fetais foram a ciclofosfamida, o 5-fluorouracil e a doxorrubicina ${ }^{1}$. Os antagonistas do ácido fólico (metotrexato) estão relacionados com anormalidades fetais quando administrados no primeiro trimestre, porém, quando prescritos no segundo e terceiro trimestres não foram associados a alterações fetais. Como ainda existem dúvidas com relação ao metabolismo do metotrexato na presença do "terceiro espaço", a maioria dos autores prefere contra-indicar o uso desta droga durante a gravide $z^{3,17,18}$. Com relação à programação do parto, deverão transcorrer ao menos três semanas entre a última dose de quimioterapia e o parto, sendo que esta medida visa diminuir as chances do recém-nascido de apresentar neutropenia e alopecia.

As pacientes estudadas em nossa série de casos foram todas submetidas à quimioterapia com o 5-fluorouracil, a epirrubicina e a ciclofosfamida, sendo que 7 pacientes $(46,7 \%)$ encontravam-se no período gestacional. Os recém-nascidos que foram expostos a ação de quimioterápicos durante a gravidez foram avaliados por neonatologistas e não apresentaram malformações.

A radioterapia é contra-indicada durante o período gestacional, sendo que a cirurgia conservadora isolada deve ser a opção, quando poder-seá aguardar até a realização do parto, para utilização da radioterapia. Com a técnica para o tratamento do câncer de mama no qual se utiliza irradiação corporal total seguida por dose de reforço no leito tumoral, o feto sofrerá dose de irradiação inaceitável ${ }^{17}$.

$\mathrm{O}$ aborto não deve ser considerado como medida integrante da terapêutica contra o câncer de mama associado à gravidez. Alguns estudos retrospectivos publicados não mostraram aumento da sobrevida nas pacientes submetidas ao aborto ${ }^{9,10}$. O aborto terapêutico deve ser recomendado naquelas pacientes nas quais o problema reside nos danos fetais que serão induzidos pelo tratamento adjuvante, como radioterapia e quimioterapia. Mas a decisão da prática do aborto nestas pacientes deve sempre ser multidisciplinar, com todo o apoio social e psicológico para a paciente e sua família ${ }^{1,15}$.

O valor exato da identificação dos receptores dos hormônios esteróides de câncer de mama 
em mulheres grávidas não está bem estabelecido. Os exames disponíveis e que são utilizados de rotina dependem da disponibilidade do receptor livre (não ligado) para fixação dos ligantes (sem técnica de permuta). Durante a gravidez, todos os locais de fixação já podem estar ocupados pelo hormônio endógeno, dando a falsa impressão de que o câncer de mama durante a gravidez apresenta receptores negativos ${ }^{18}$. Os nossos dados são correspondentes aos da literatura, em que 60\% das pacientes apresentaram receptor de estrógeno negativo pelo estudo imuno-histoquímico, o qual avalia a expressão da proteína receptora responsável pela interação entre a célula neoplásica e o hormônio estrogênico.

Os primeiros relatos acerca do câncer de mama em mulheres grávidas dão a impressão de que a gravidez subseqüente ao tratamento da doença poderia ter um efeito protetor à mulher, pois elas apresentavam intervalo livre da doença maior. Reavaliando estes relatos, conclui-se que esta maior sobrevida estava relacionada com a história natural do câncer de mama, pois mulheres que apresentavam estádio clínico precoce na época do diagnóstico eram as mesmas que engravidavam. As mulheres que apresentavam estádio clínico avançado na época do diagnóstico não tinham oportunidade para engravidar ${ }^{19}$.

Existe muita controvérsia quanto ao tempo necessário para que a paciente possa engravidar. Alguns autores recomendam três anos ou até cinco anos, apenas baseando-se no período com maior probabilidade de recidiva ${ }^{1-3}$. Mas estes números nos parecem cabalísticos e não existe nenhuma base científica para estipular este intervalo. Finalmente, não poderíamos nos furtar de enfatizar a necessidade, nas pacientes que tiveram câncer de mama e estão em tratamento adjuvante, da anticoncepção que deve ser realizada com dispositivo intra-uterino, métodos de barreira ou associação dos métodos não hormonais. Assim, recomendam-se, tendo em vista o aumento de gravidezes nas mulheres na terceira e quarta década, exame clínico adequado e exames de imagem, sendo a USG para mulheres com idade inferior a 35 anos e a mamografia para mulheres com idade acima de 35 anos na fase pré-concepcional. Devese aprimorar o exame clínico nas primeiras consultas pré-natais, pois neste período encontrarse-á mais facilidade que nos demais trimestres da gravidez, tendo em vista o aumento da hipertrofia e do ingurgitamento mamário. Qualquer nódulo que for detectado durante a gravidez não deve ter postergada a sua investigação clínica, radiológica e cito-histológica. E por fim, na relação médico-paciente, dar segurança à paciente com câncer de mama associado à gravidez e a sua família, informando que o tratamento do câncer de mama durante a gravidez é seguro, o abortamento não melhora o prognóstico, e sempre enfatizar que o tratamento deve ser realizado por equipe multidisciplinar, para melhores resultados clínico e psicossocial.

\section{ABSTRACT}

Purpose: to report 15 breast cancer cases associated with pregnancy and to compare to a control group with breast ductal infiltrating carcinoma, evaluating clinical staging, metastatic axillary lymph node involvement, histopathologic aspects related to nuclear grade, histology grade and estrogen and progesterone hormonal receptors.

Method: a retrospective study of 15 cases of patients with breast cancer associated with pregnancy, attended at Mastology Department in the Woman Health Reference Center, Pérola Byington Hospital, São Paulo, was done between September 1996 and April 2001. The evaluation of clinical staging, time of diagnosis and involved axillary lymph nodes was the main study basis. Also age, parity, histologic type, applied treatment, histologic characteristics regarding nuclear grade and histologic grade and the presence of hormonal receptors in the tumors were analyzed.

Results: we observed that 7 patients $(46.7 \%)$ presented a locally advanced breast cancer (clinical stage IIIA and IIIB) and that 3 patients (20\%) presented a disseminated disease at the moment of diagnosis. The patients presented on average 2.4 involved axillary lymph nodes and in only one patient the lymph nodes were free of disease (6.6\%). Regarding time of diagnosis, $40 \%$ of the tumors were diagnosed during the lactational period, $46.7 \%$ during the second trimester and $13.3 \%$ during the third trimester. The pregnant patients were compared to a control group of non-pregnant patients in the same age range, all of them with infiltrating breast carcinoma, and clinical staging, axillary lymph node involvement, nuclear grade, histologic grade and estrogen and progesterone hormonal receptors were evaluated. There was a statistically significant difference $(p=0.0022)$ regarding clinical staging and axillary lymph node involvement $(p=0.0017)$, and no statistically significant difference as concerns the remaining parameters.

Conclusion: breast cancer associated with pregnancy is a neoplasia with a bad prognosis. There is no difference when comparing pregnant patients with non-pregnant patients in the same age range, the advanced clinical staging at the moment of diagnosis being the determinant factor for survival.

KEY WORDS: Breast: neoplasms. Pregnancy complications. Chemotherapy. 


\section{Referências}

1. Berry DL, Theriault RL, Holmes FA, et al. Management of breast cancer during pregnancy using a standardized protocol. J Clin Oncol 1999; 17:855-861.

2. Gemignani ML, Petrek JA, Borgen PI. Breast cancer and pregnancy. Surg Clin North Am 1999 ;79:115769.

3. Brewster WR, DiSaia PJ. Breast cancer associated with pregnancy. In: Winchester DJ, Winchester DP, editors. American Cancer Society. Atlas of Clinical Oncology - Breast Cancer. London: Hamilton. 2000. p.258-9.

4. Kilgore AR, Bloodgood JC. Tumors and tumor-like lesions of the breast in association with pregnancy. Arch Surg 1929; 18:2079-98.

5. White TT. Carcinoma of the breast and pregnancy. Ann Surg 1954; 139:9-18.

6. Haagensen CD, Stout AP. Carcinoma of the breast: carcinoma of operability. Ann Surg 1943; 118:85970 .

7. Harrington SW. Carcinoma of the breast: results of surgical treatement when the carcinoma occurred in course of pregnancy or lactation and when pregnancy occurred subsequent to operation, 19101933. Ann Surg 1937; 106:690.

8. Gupta RK. The diagnostic aspiration cytodiagnosis of breast masses in association with pregnancy and lactation with an emphasis on clinical decision making. Breast J 1997; 3:131-4.

9. Giacalone PL, Laffargue F, Benos P. Chemotherapy for breast carcinoma during pregnancy: A French national survey. Cancer 1999; 86:2266-72.
10.Moore HCF, Foster RS. Breast Cancer and pregnancy. Semin Oncol 2000; 27:646-53.

11.Bernik SF, Bernik TR, Whooley BP, Wallack MK. Carcinoma of the breast during pregnancy: a review and update on treatment options. Surg Oncol 1998; $7: 45-9$.

12.Zemlickis D, Lishner M, Degendofer P, Panzarella T, Sutcliffe SB, Koren G. Fetal outcome after in utero exposure to cancer chemotherapy. Arch Intern Med 1992; 152:573-6.

13.Zemlickis D, Lishner M, Degendofer P, et al Maternal and fetal outcome after breast cancer in pregnancy. Am J Obst Gynecol 1992; 166:781-4.

14.Gwyn K, Theriault R. Breast Cancer during pregnancy. Oncol 2001; 15:39-46.

15.Liberman L, Giess CS, Dershaw DD, Deutch BM, Petrek JA. Imaging of pregnancy associated breast cancer. Radiology 1994; 191:245-8.

16.Applewhite RR, Smith LR, DeVicenti F. Carcinoma of the breast associated with pregnancy and lactation. Am Surg 1973; 39:101-4.

17.Lichter AS, Lippman ME. Special situations in the treatment of the breast cancer. In Lippman ME, Lichter AS, Danfoth DN, editors: Diagnosis and Management of the breast cancer. Philadelphia: WB Saunders; 1988. p. 414-38.

18.Sakai F, Saez S. Existence of receptors bound to endogenous estradiol in breast cancers of premenopausal and postmenopausal women. Steroids 1976; 27:99-110.

19.Di Saia Ph J, Creasman WT. Clinical Gynaecological Oncology. $3^{\text {rd }}$ edition St Loius: C V Mosby; 1989.p 511.

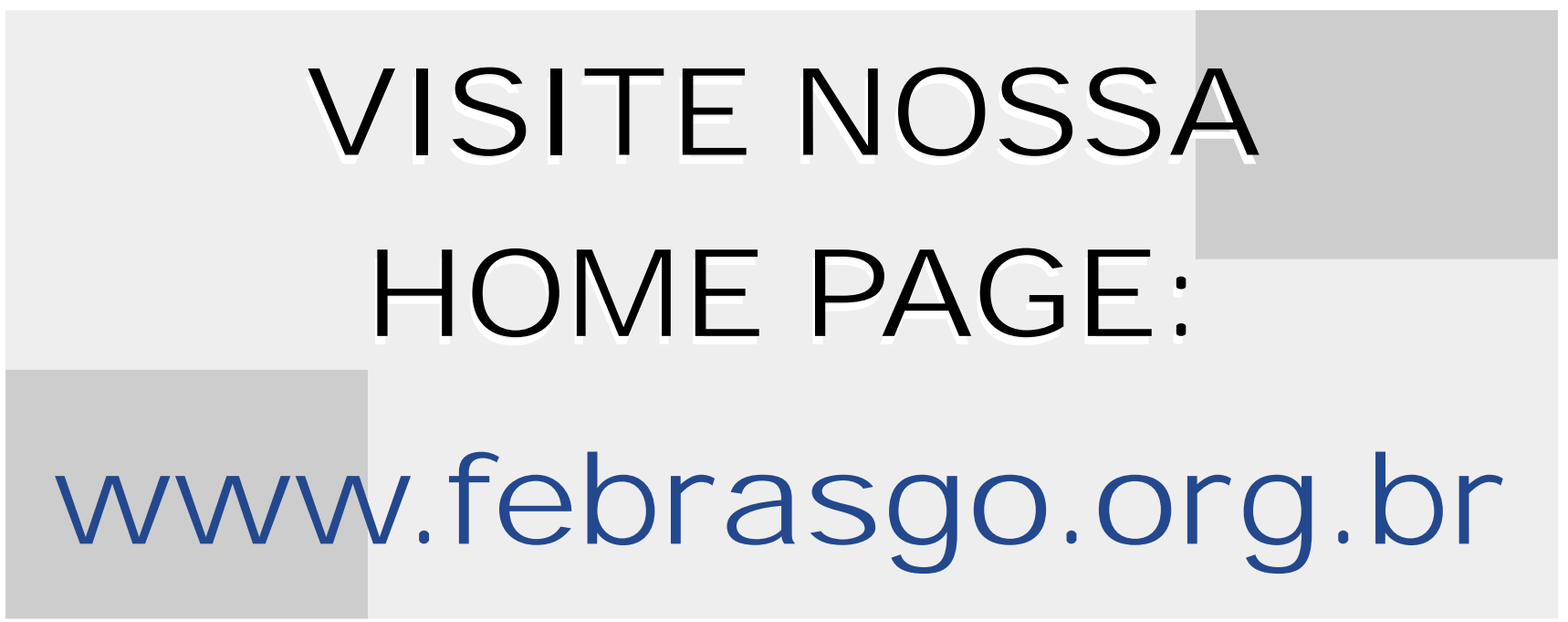

